\title{
Automotive Systems Engineering
}

\author{
Jae Kyun Chang*. \\ * CARNES Co. Ltd, Anyang-city, Gyeonggi-do , \\ Korea (Tel: 031-389-0312; e-mail: jaekyun.chang@carnes.co.kr).
}

\begin{abstract}
Automotive Systems engineering is the key technology for many innovations in vehicle construction. In Vehicle development, quality control, risk and cost management is an important precondition for successful and on-time projects, in addition to expertise in increasing complexity. To realize increasing demands concerning safety, economic impact, fuel consumption, comfort and high quality a coordinated and systematic development process is essential in this contribution. Based on functional and non functional requirements, an open and modular system architecture must be designed. This system architecture, as the basis for the system design and implementation, support aspects like re-use, function partitioning, scalability and distributed development with well defined system interfaces. Furthermore, a hierarchical decomposition of the system into sub system and the necessity to realize a system by using different technical and physical principles will be the main topics out of system engineering and architecture design.
\end{abstract}

\section{INTRODUCTION}

The last 10 years of automotive industry have brought many kind of innovation which concentrated in advanced technology and its smart application. For many type of product, the concept of "local" market is disappearing. Engineers increasingly work in distributed companies, connected by e-mail to colleagues who may be thousands of miles away. The driving factors to be controlled in automotive industry are:

- $\quad$ increased complexity of products;

- globalization of marketplace;

- reduction of product development cycle;

- $\quad$ software as dominant force for change in almost all new products;

- $\quad$ worldwide deployment of new technology

- reuse of components;

- $\quad$ partnership with other leading company;

To manage this increasing complexity, to meet the market requirements and to cope with changing environments, System engineering is the key technology to be realized and applied in the development of automotive products which can be derived out of system engineering as system components, and effective system development processes are the key intellectual assets for a modern project control and success.

\section{USER REQUIREMENT}

The first step of system engineering is the analysis of market trends and to define the end users needs and requirements, followed by designing and developing the initial product to meet the user requirement and to get the market acceptance. The role of capturing and managing requirements-sometimes we call requirement engineering- helps us to define what the product should perform at the end. And then the architectural design should define what will be produced. The initial systems will be generated through system development process and as next step, system components will be transferred to mass-production, for further deployment, and operational use. Feedback from end users and market response, then determines the shape of future products. For many businesses, the key success factor is delivering the right product to market before competitors, and even the development cost is secondary product timeliness. If the product is good enough and well accepted by end user, then take the whole of the market. This puts a huge premium on being in advance of rivals. Typically, $50-75 \%$ of development time is spent on early pre-production phases. The front-end processes of requirements and design - so called front-loaded system engineering - is very important phase in total development duration. This is where we have to look into, how the product can be developed for "time to market". Supplying better products and shortening the development time therefore demand dramatic improvements in requirements engineering, system design approaches and system development process.

\section{SYSTEM REQUIREMENT}

The two most common reasons for project failure were incomplete requirements and lack of user involvement. These lead fail to deliver the right product on time and within the budget. Explain it in more positive way, the key factors for success are well planned front-loaded system engineering processes, based on understanding of the market and involving end user requirements. System engineering is about creating solutions to problems, and managing the technical complexity of the resulting developments. It is 
creative activity, defining the requirements and realizing it into the product which to be built in the vehicle. The system engineering role is to handle whole vehicle life cycle in a balanced way. During whole and each development phase, system engineering results is to be shown through trade-off between competing factors such as performance, risk and cost. The system engineer should always monitor and control the development status for the potential risk from end-to-end, confirming decisions only when the risks are acceptable and manageable. Capturing and managing requirements is a vital system engineering task. Requirements are the foundation for any system product. Well performed system engineering brings the success at the end of the system development project. System engineering is different from specialist disciplines such as mechanical or electrical engineering. Technical coordination between all related development areas is a core element of its work. All the system engineering results will be created, documented and managed for changes and variants with systematic control of Engineering DB system.

\section{CREATION OF SYSTEM ARCHITECTURE}

System architectural design defines clearly what is to be built, at the end in the vehicle. This is potentially the most creative part of the system engineering process. When it is complete, each system component can be seen separately by the responsible engineering group and developed for mass production with selected supplier. It transforms system requirement into more concrete form, by allocating functions to hardware, software or defining interfaces and communications. The architecture should compact enough to let design options be explored and optimized, before going into the detail of the component design. Architectural design optimizes the design characteristics to satisfy the requirements as far as system target is met, in functionality, quality and cost. The objectives for the system architectural design stages are:

- Creation of architecture that fit to user and system requirements with operational environment

- Definition of the system components to be built in vehicle

- Definition of how system components interact

- Trade-off between alternative architectures to maximize the system effectiveness

- Partitioning of system components

- Definition of the deliverable items

- Estimation of cost and countermeasures for risks

Interfaces in system architectural design must be clear, stable, and decoupled at the end.

Partitioning and integration of system functions is the essential issues in system architectural design which lead at the end, vehicle optimization from system level to component level. Cross functional integration between sub-systems and domains such as Power train, chassis, Body, Electronics etc. are also valuable to optimize the whole vehicle architecture. Pre-condition for creating successful system architecture is not only the job sharing between different domains or departments but also cooperation of all related domains and departments: communications and common decisions are essential.

\section{SYSTEM INTEGRATION}

The starting point for system integration and verification is release of components from the suppliers. Configuration and quality checks are needed to ensure the right parts have been supplied. Individual components are tested, before being integrated to produce higher level sub-systems or assemblies. Integration and verification can sometimes be a frustrating process, made more difficult by being well separated from the requirements and design stages. For this reason, system integration and verification engineers should ideally be involved from the initial requirements stages. System integration and verification is always a compromise, balancing the rigor of testing against the resources and time needed to perform it. Testing everything would take too long time and too expensive. Tests therefore have to be designed for effective fault detection. In the test process, the procedures are running against the components, checking the results against what was expected. In producing a complex system product, system integration and verification may be repeated several times. Firstly, the process certifies that the design is satisfactory by testing the development models, giving clearance to manufacture operational units (design verification). When the production units are built, a similar process tests them to certify that the manufacturing process is working satisfactorily (production verification). As a system engineering aspect, verification should be done in each system level of development.

\section{SYSTEM PROJECT MANAGEMENT}

System project management and system engineering are highly inter-dependent, because every technical decision has a management consequence and vice-versa. System engineering defines the deliverables; there are the objects handled by project management and which come together to satisfy the requirements and integrate to form a complete system. The project management information involves costs, schedule and quality and work packages, all are attached to the same deliverable structure.

Perhaps the cost differences between two disciplines lies in the fact that the system engineering role is responsible for creating, defining and improving the product. This involves definition of requirements and architecture of the system, so that cost estimation and risk management role is more concerned with the delivery of that system on time and to schedule.

The system project manager has the responsibility for planning and controlling the following tasks:

- Leading the system project and creating a good working atmosphere 
- $\quad$ Planning the system projects : process definition and preparation of method and tools

- $\quad$ Managing all the elements outside the project

$$
-
$$

Configuration management is the foundation for project management and the tie back to system engineering. It helps co-ordinate the work and ensure reproducibility of any system state. Configuration management is sometimes thought of in mechanistic terms, but the benefits are so high it should be handled carefully. The discipline ties system engineering to project management. Configuration management enables work to be efficiently partitioned to different individuals and organizations. Therefore configuration and change management tools are used company wide to secure this important issue.

To shorten the total development time, system development is performed as parallel development of system components (simultaneous engineering). This means in the system function development, parallel application and tests of the function must be performed at the same time frame with the changes of each component, this is one of important issues to be controlled under system project management.

The base of common understanding for the new system function is made very oft with graphic function model. This kind of development method brings also other advantages. The specification is very clear to be defined without any kind of specific contradiction. The specification is in the PC applicable (running as simulation) and can be experienced in the vehicle (via proto typing). Due to this advantage, this digital draft of specification is very rapidly extended in the automotive electronic developments. To get this development environment and strict application, system project management is interested on this kind of approaches from beginning of system project. Standardized simulation tools can be used to evaluate the new function in early phase and based on that, software code can be generated automatically. This will support system engineer to evaluate and to decide in early phase.

At the end of the system project, quality, risk and cost is evaluated and continuously monitored for further improvement and successful project.

\section{SYSTEM AND SOFTWARE}

All new systems now contain large amounts of software. Most of the new innovation in automotive electronics and added value are implemented through software. A modern car may contain over than 50 microprocessors, which used for controlling the performances of such as engine control, pollution control, security control, control of air-conditioning, realization of infortainment and also providing convenient functions. In most of system development case, software is also the component on the critical path, and takes the blame for system development problems. The fundamental reasons for this have little to do with software itself, but are related to development size and complexity to be defined and how the function is specified before software development is started. Modern system products are more complex than before, and most of that complexity is realized by software. The software engineering takes the package of requirements from the level above and implements it as a piece of tested software, ready for integration.

As software grows more complex, it behaves more like a system and has to be managed as a system engineering problem. At the management level, however, the development processes for software and systems are similar. The software is so important, pervasive and time-critical in almost all modern systems that it must be as a key-level issue, despite the fact that the individual software components are developed at a lower level. In turn this requires well-defined systems engineering processes which show how to structure the software within systems. The software and system development processes must be consistent across the project. As a example or guide line for the software development process, CMM, CMMI or SPICE are applied, like as system engineering, for this reason proper system and software development tools are used to keep in line with predefined processes.

\section{INFORMATION CONTROL}

System engineering results such as system specifications, system development related information is now increasingly handled in a database, like as DOORS and project management supporting tools. The system processes provide the starting point for the information model, before it is tailored; the act of tailoring the information model is equivalent to modifying the process.

To summarize the important objectives in handling information are;

- creating and writing information once;

- $\quad$ using same information many times

- $\quad$ finding information easily

- naming information by convention

- $\quad$ selecting subset of information

- detecting missing information

The traditional approach of control through paper-based documents requires super-human discipline. The key trends are to replace the system information electronically and to organize and control that database through the rules defined for the organizational processes.

\section{CONTINUOS IMPROVING SYSTEM ENGINEERING PROCESS}

In many industry areas, the system engineering processes are currently fragmented or non-existent, despite valiant attempts from individual enthusiasts. Many companies spend a fortune on $\mathrm{R} \& \mathrm{D}$ and product development, but are reluctant to allocate a fraction of that on improving their development process. However, many companies are trying to improve 
their internal processes. Enhancing systems engineering across the company is a process not on event that involves far more than just buying tools, and the real investment is in improving the discipline. The success factors for process improvement are;

- defined and measurable objectives

- generate the bridge the gap between ideal and current

- $\quad$ create a good process for product management

- $\quad$ previous successful experience in front-loaded system engineering processes

- management commitment

- $\quad$ trying to external certification, such as ISO, QS CMMI, and so on.

The cost of improving the systems engineering processes must be justified in business terms, taking into account the current costs of 'non-quality'. When a product fails, it must be replaced or repaired, leading to extra resources for documenting the faults, handling the problems and transportation, etc. Producer should apologize to the customer, but it can not be recovered the loss to its reputation. Making the business case with the cost for improving the systems shows that Potential savings $=$ The current costs of non-quality or non-performance should be justified before.

Benefits from improvement of system engineering process can deliver the following:

- improved time to market

- more satisfied customers

- $\quad$ earlier delivery of an operational product

- control over the development

- a measurable process

- a process where progress is visible to management

Costs that apply across multiple projects should be borne by the business. These include costs in developing standards and training materials and so on.

\section{CONCLUSION}

Automotive system engineering embraces the following concepts;

- a balanced approach ; the system and its environment should be considered as an entity. Trade-off between requirements, costs, and timescales are an essential part of the work.

- Creativity and domain knowledge: system engineering demands intense creativity, particularly in the system requirements and architectural design processes.

- Coping with risk and change: risk and human failures are constants in system development and operations. Although system engineering is trying to make prediction and direction, the future is only partially discernible. We can't anticipate every problem, but we must build systems which can cope with change.

- $\quad$ Managing complexity: Process and tool can help contain the problems which result from this complexity, both in product and development process.

- $\quad$ Re-using experience: "Lesson learned" from the past should be reviewed and applied for running project.

- A end user driven approach: end users are the experts at using the system. We have to build systems which satisfy them. Requirement management is therefore a key for the system engineering.

- Whole life cycle consideration

- Comprehension of multiple disciplines

- Improving the performance of the team

- Linkage to the business goal

Out of successful automotive system engineering we can get experiences and set directions in;

- Standardized system function

- $\quad$ Tailored efficient test concept

- Harmonized architecture for vehicle and sub system, and for system components

- System \& Technology roadmap to fit future trends

- Platform approaches in System and System Component, even in technical domains, Hardware, Software etc.

The development of leading automotive system is one of the most challenging activities, and driven by a need to cope with complexity. System engineers aim to take intricate problems and produce robust practical solutions.

Almost of all industry understand now the needs of improving their development process. Systems engineering is crucial to the future of any customer or supplier organization.

The world is moving rapidly to a knowledge-based economy, and the really successful economies will be those that manage their knowledge effectively.

System engineering is the core technology to create value from the situation - it closes the loop between customers and developers. Business and countries that cannot understand it will disappear from complex product development.

\section{REFERENCES}

Max. L. J. Wolf, Rudolf Mlekusch ; Projektmanagement live, Expert verlag.

R. Stevens, P. Brook, K. Jackson, S. Arnold ; systems engineering, Prentice Hall, Pearson Education 1998

Internal document of company CARNES ; systems Engineering procedure, automotive electric and electronic system development. 